\title{
Generalized Electronic Interviewing System (GEIS): A program and scripting method for conducting interviews in multiple modes
}

\author{
ROSS CORKREY \\ University of Aberdeen, Aberdeen, Scotland \\ and \\ LYNNE PARKINSON \\ University of Newcastle, Newcastle, New South Wales, Australia
}

\begin{abstract}
A program called the Generalized Electronic Interviewing System (GEIS) was developed for conducting interviews, using computer-assisted telephone interview (CATI) and interactive voice response (IVR) modes without the need for a programmed interface. GEIS questionnaires were prepared using a common script syntax in all supported modes. Scripted development allowed for rapid interview development without the need for programming. A GEIS script specified the following: question texts, including variable texts; answer option texts; numeric codes for answers; range check information; logical question-branching information; interview status information; do-loop information; and IVR information, such as key codes and voice messages. GEIS thoroughly checked scripts for logical or syntactical errors. GEIS required SAS Version 8.0, and survey data were accumulated within SAS data sets. An application of GEIS to conduct a survey involving CATI, IVR, and a combined hybrid method is described. The CATI results deviated in the direction expected for sensitive questions, whereas IVR obtained a small sample size, rendering the results unreliable. However, the hybrid method was found to provide more accurate telephone survey data on alcohol consumption than did CATI alone. The program may be downloaded from the Psychonomic Society Web archive at www.psychonomic.org/archive/.
\end{abstract}

In previous work, this research team found that interactive voice response (IVR) showed promise as a survey and research tool but was yet to be fully explored (Corkrey \& Parkinson, 2002b). Other commonly used modes of data collection include computer-assisted telephone interview (CATI; Nicholls, 1988), paper questionnaires (Cannell \& Fowler, 1963), and touch screen computers (Kissinger et al., 1999).

The potential scope for research projects will expand as additional communication methods appear. These may include mobile telephones (Alemagno et al., 1996), short message service (Hansen \& Dørup, 2001), Web pages (Couper, Traugott, \& Lamias, 2001; Smith, 1997), and e-mail (Schaefer \& Dillman, 1998; Sheehan \& Hoy, 1999).

Currently, interview scripts are often prepared by common office software, specialized telephony programs, or in-house custom applications. For example, a paper questionnaire may be prepared using a word processor. Such methods may not provide any means of checking the logic structure of the instrument and may not provide

This project was funded by grants from the Hunter Medical Research Institute and the University of Newcastle and formed part of the doctoral studies of R.C., which were supported by an Australian Postgraduate Scholarship. Correspondence concerning this article should be addressed to R. Corkrey, School of Biological Sciences, University of Aberdeen, Lighthouse Field Station, George Street, Cromarty, Ross-shire IV11 8YJ, Scotland (e-mail: r.corkrey@abdn.ac.uk). consistency, so that documents will differ in style and content between research teams, and the software used may incorporate programming errors that can be difficult to disentangle from questionnaire errors. In addition, the expanding range of communication methods may be challenging for limited research budgets and may reduce comparability between modes.

As new communication methods arise, they are quickly adopted by researchers in order to exploit their new features and advantages. In order to maintain comparability of results over time, a uniform approach to interview specification is required. For example, an interview script for a paper questionnaire should be transferable to a telephone interview with minimal changes. The script should be supported by a single well-tested program, so that any errors remaining can be attributed to the questionnaire. As new communication methods appear, the supporting software may be modified, but the script should ideally remain constant, although the reliability and validity of the modes will need assessment. Scripts also serve as project documentation. We suggest that these requirements are best achieved by an open-scripting and opensource software (Hansen \& Dørup, 2001), avoiding proprietary formats that may be restrictive, dependence on programs that eventually become obsolete, and rapid elimination of programming errors (da-Silva, Rodrigues, Leite, \& Milan, 2003). 
As a first step, a program was written, the Generalized Electronic Interviewing System (GEIS), to create and run electronic interviews without the need for programming that could introduce new errors with each survey. Instead, it uses a flexible and open-scripting method. Open source is used to encourage the program's further development or to allow it to be adapted to local needs. GEIS allows a single script format to be used in multiple modes without the need for programming, resulting in rapid survey development.

Currently, GEIS supports the CATI and IVR modes. In CATI surveys, a computer is used to assist the telephone interviewer by ordering and presenting questions to be asked and then recording the responses (Nicholls, 1988). In IVR, the human speaker is replaced by a highquality recorded interactive script to which the respondent provides answers by pressing the keys of a touch telephone (touchphone). It differs from a CATI by lacking an interviewer to read questions and enter the answers into the computer. Many attributes of CATI are shared by IVR, including the following listed by Nicholls: automatic logical skipping or branching based on answers to earlier questions; randomized option and question order; interviews that can be interrupted and resumed at a later time; single-choice as well as open-ended responses to questions; validation of responses as they are entered; provision of feedback on inappropriate responses; call scheduling; and automatic interview and recording keeping. In addition, IVR has the potential for obtaining a reduced response bias when used to ask sensitive questions (Corkrey \& Parkinson, 2002b).

GEIS also provides an efficient means of combining modes in ways that would otherwise be difficult to achieve, due to the programming complexity involved. It is possible to combine CATI and IVR so that the two modes are used to conduct different parts of an interview. This can allow the more sensitive questions to be asked by the more anonymous mode. GEIS has some support for touchscreen interviews, but only CATI, IVR, and their combinations are discussed in this article.

An example is given in this article that illustrates the use of CATI, IVR, and a combination of the two. The results are compared with those of an anonymous paper questionnaire method.

\section{Aims}

The aims of this article are to describe (1) the use of GEIS to create an interview, (2) the implementation of GEIS, (3) the storage of data within GEIS, (4) GEIS scripts, and (5) an application of GEIS.

\section{Using GEIS to Create an Interview}

The overall procedure for developing an interview in GEIS is shown in Figure 1. An interview script was prepared, using a text editor, and was imported into GEIS. Errors in syntax were reported by GEIS at this stage. These were corrected, and the script import attempt was

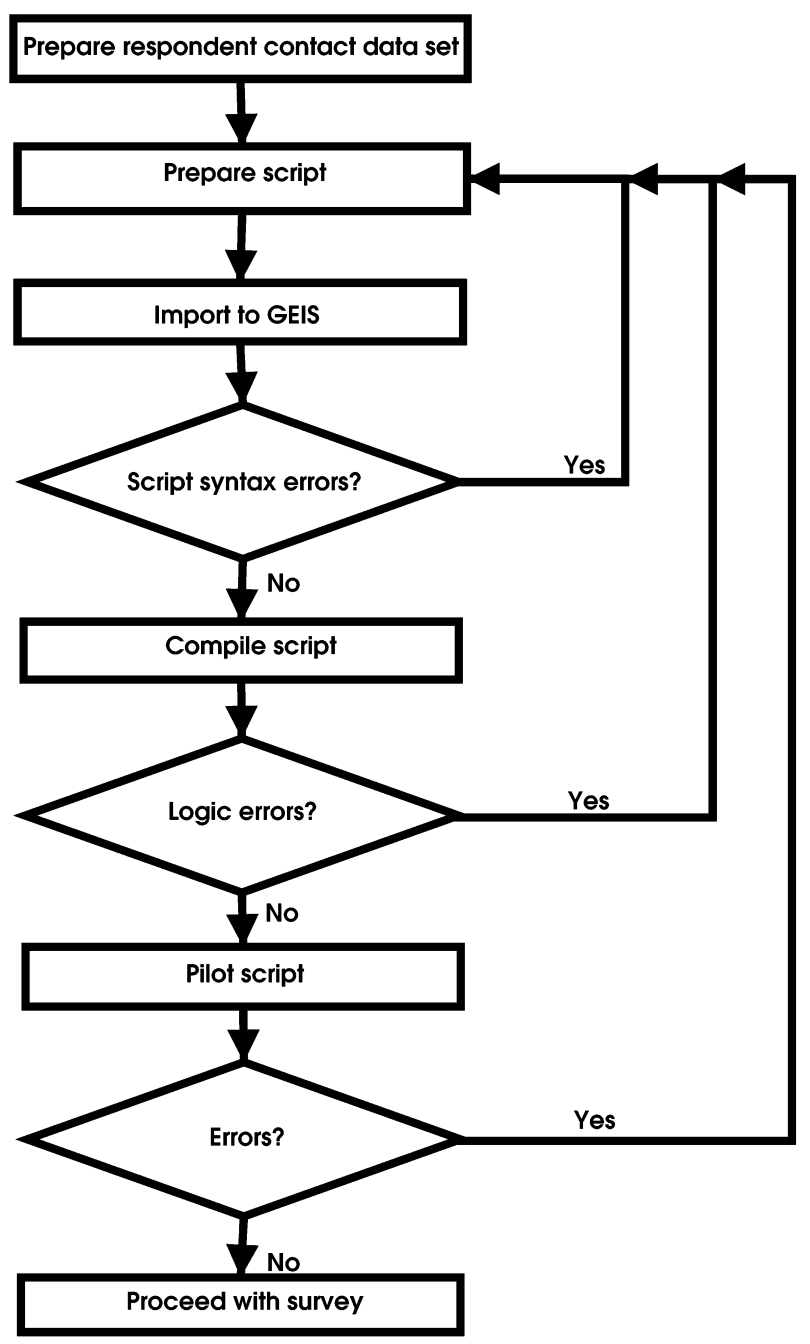

Figure 1. The procedure for developing a script within GEIS.

repeated until no errors occurred. In the next stage, termed compilation, GEIS conducted detailed checks to ensure that all the script items were validly defined, interview control information existed, it was possible to jump from each item to another, and every item could be reached from an earlier one. The checks performed were very detailed, so that most scripts that passed compilation ran reliably on the first attempt, resulting in a very rapid interview development.

The script was then piloted by the survey designer and volunteers to find any errors not detected by GEIS, refine the wording of questions, and train interviewers. Once script piloting had been completed, the project data sets were uploaded to a share server (SAS Institute Inc., 1991), described below. The share server allowed respondent records to be individually accessed by CATI interviewers or an IVR system. A typical hardware arrangement is illustrated in Figure 2. 


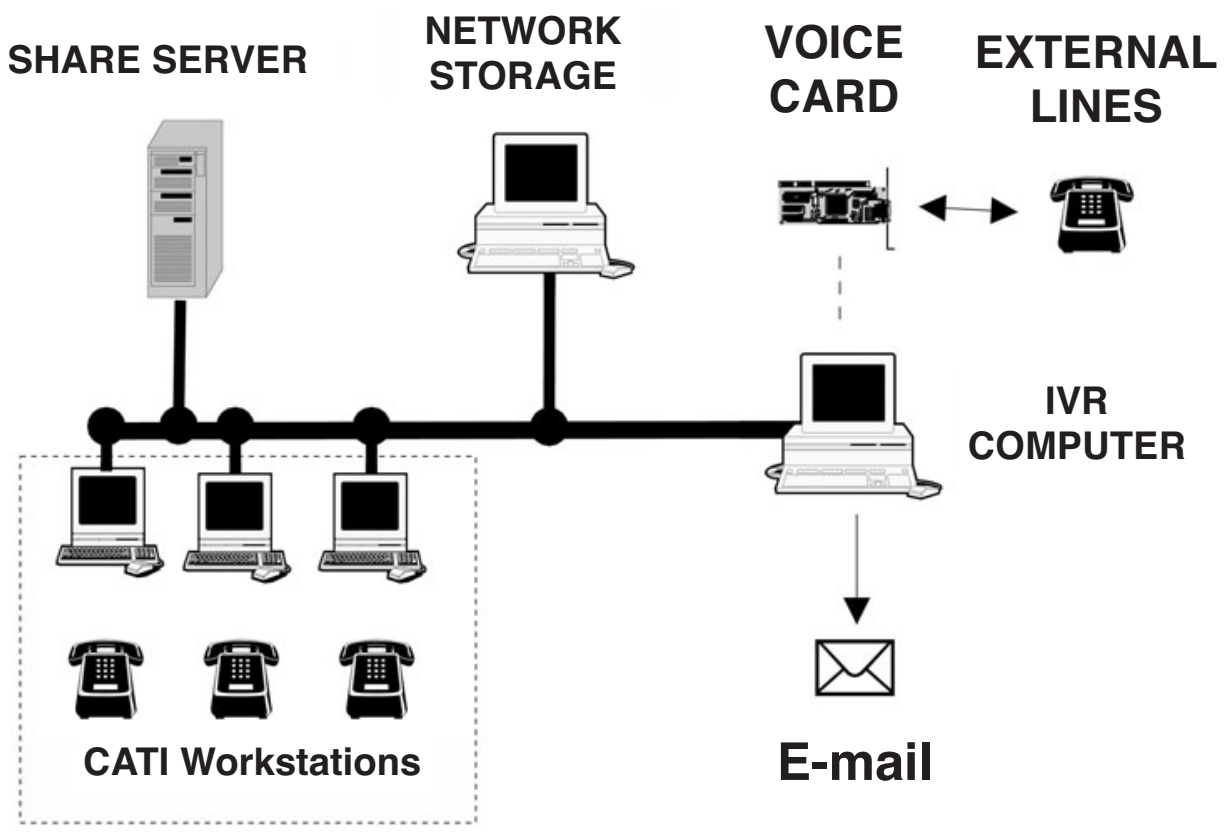

Figure 2. Typical GEIS hardware arrangements.

\section{Implementation of GEIS}

Running GEIS required SAS software (SAS Institute Inc., 1999) and Visual Voice Pro Version 5.0 software (Artisoft Inc., 1998), running within Microsoft Windows. It is expected that GEIS should be easily ported to SAS running within other operating systems after rewriting the graphical interfaces, but with minimal code changes. GEIS is available for SAS Version 8.0 and is expected to run with minimal changes in later versions.

Due to the portability of the SAS environment, the program should also run with minor modifications on non-Windows platforms. The portability of the IVR functionality will depend on support within the proposed platform. The use of different voice cards may also require code modification, although we have successfully tried two types of Dialogic voice cards. However, the high cost of IVR hardware prevents us from testing it more widely. However, by releasing the program as open source, we hope that others will adapt the program to a wider range of hardware. GEIS may also be ported to other non-SAS environments, with appropriate rewriting of code and graphical interfaces.

\section{The GEIS Program Windows}

GEIS consisted of a hierarchical series of windows accessible from a control panel (Figure 3) that allowed a variety of functions: script importing and editing, compilation, interview piloting, system reports, system maintenance, and data set finalization. The more important of these functions are described below.

Script importing and editing. Interview scripts were stored in text files, using any text editor or GEIS. They were then imported into GEIS. Scripts could contain links to nested subscripts, typically for the inclusion of standard question modules. A window (Figure 4) displayed the results of the script import process.

A phrases editor utility was available within GEIS for editing, playing, and recording IVR messages. The editor could play sound files but could not edit them. The editor displayed the text for each question to allow a recording to be made and edited with a suitable external program. GEIS does not provide a facility to edit sound files directly, since this is done better by more specialized programs. Once a recording had been made, the editor was used to assign the recording filename to the script item and assign a keyword to the recording. The keywords were used within the script to specify which recordings to play.

Script compiling. Compilation consisted of creating survey data sets and extensively checking the script for logical errors. The GEIS compiler wrote error, warning, and information messages to the GEIS internal log. After each compilation, the GEIS log was examined, and if errors were found, the script was modified and recompiled.

Interview piloting. The interviewing system could be run from the control panel to conduct pilot tests of scripts. In CATI surveys, the interviewing system consisted of windows for log-in, respondent selection, and interviewing. The log-in window was used by interviewers to $\log$ into GEIS. In the respondent selection window, respondents appeared in a pull-down list, which also allowed for searching by particular name or telephone number. The respondent interviewing window was used in both CATI and IVR (Figure 5). In order to minimize interviewer 


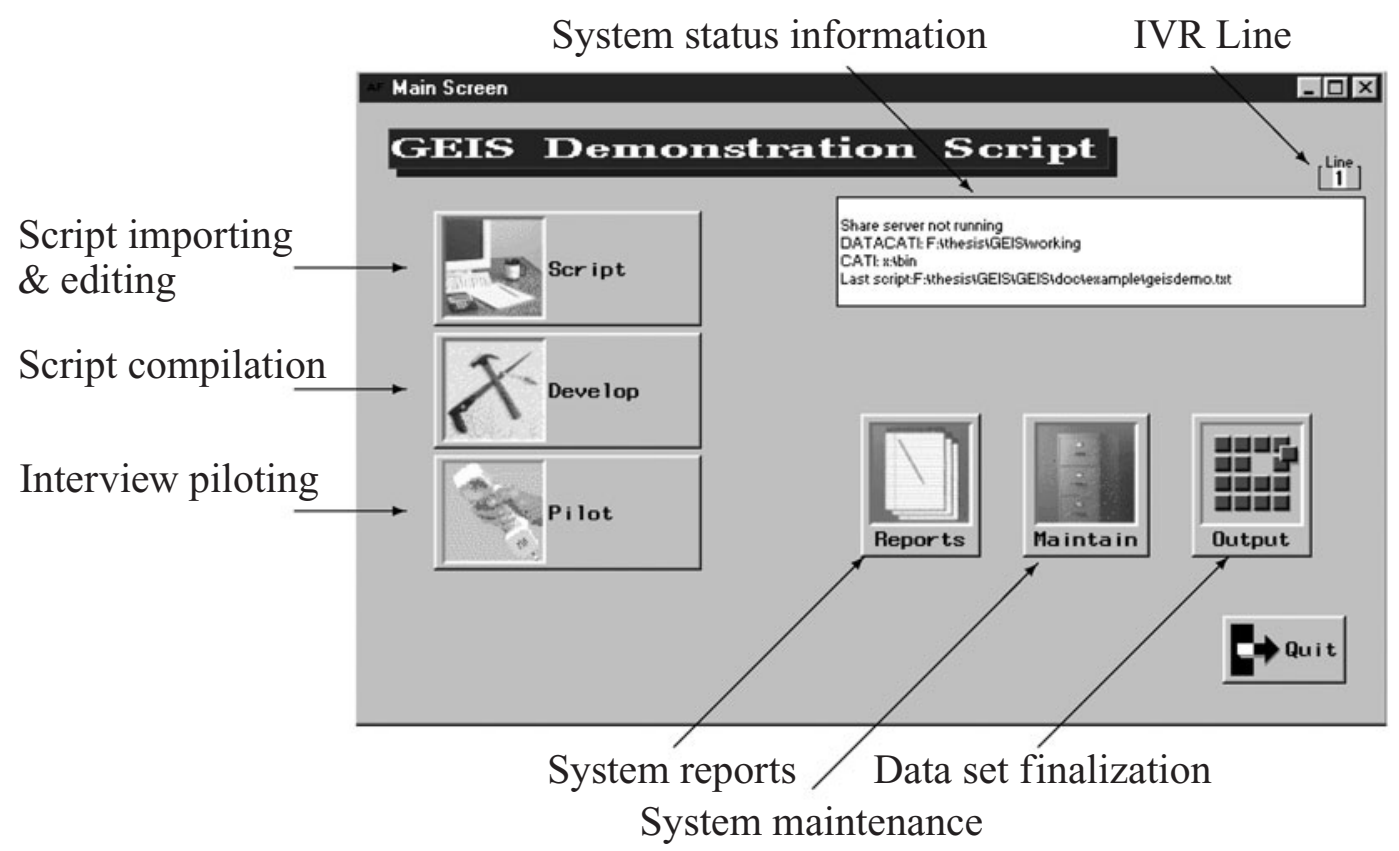

Figure 3. The GEIS control panel.

training and interview variation in CATI, the design of the interviewing window remained constant between projects. For example, the data entry controls remained in the same location on the screen for each question and survey project.

In the respondent-interviewing window, the "Next" button caused GEIS to display the next appropriate question, and the "Back" button caused GEIS to redisplay the question last asked. In the IVR mode, movement back and forward through the script was controlled by the respondent's pressing keys on his or her touchphone. The * key returned to the previous question, and the \# key moved ahead to the next question or repeated the current one.

GEIS never displayed the next question until the current question had been answered but did allow the previous

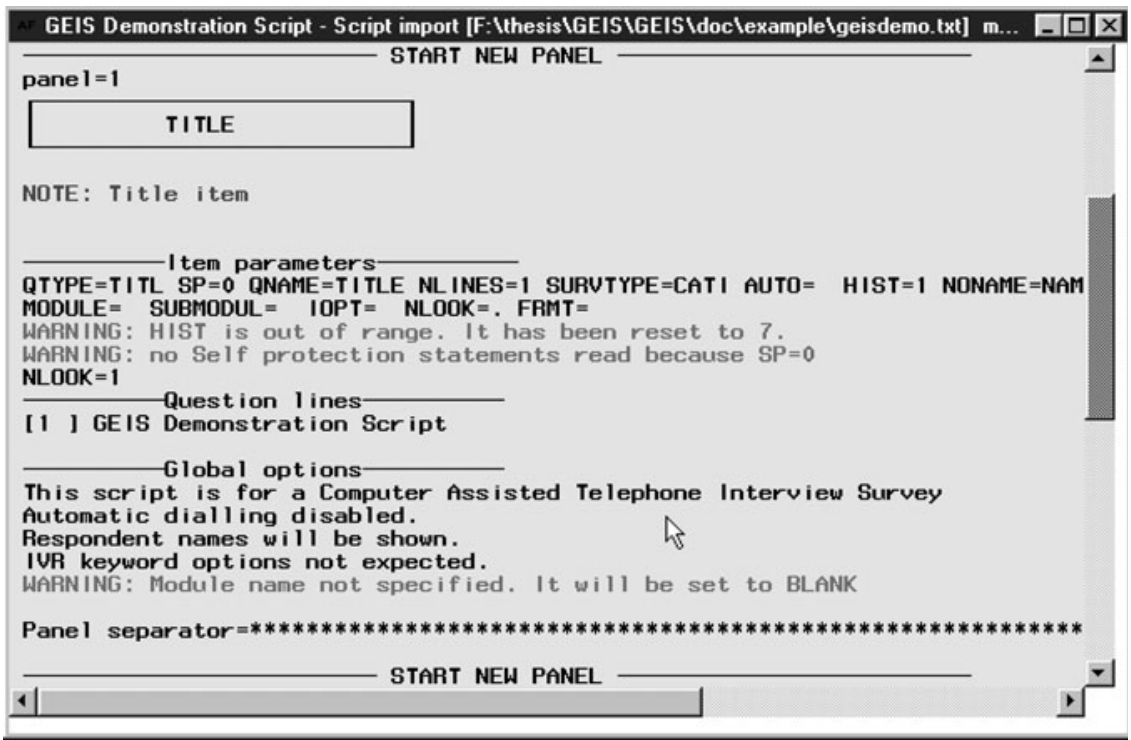

Figure 4. The GEIS script import results window. Errors and warnings were displayed in differently colored texts. The figure shows the first screen of the results of importing a script. 


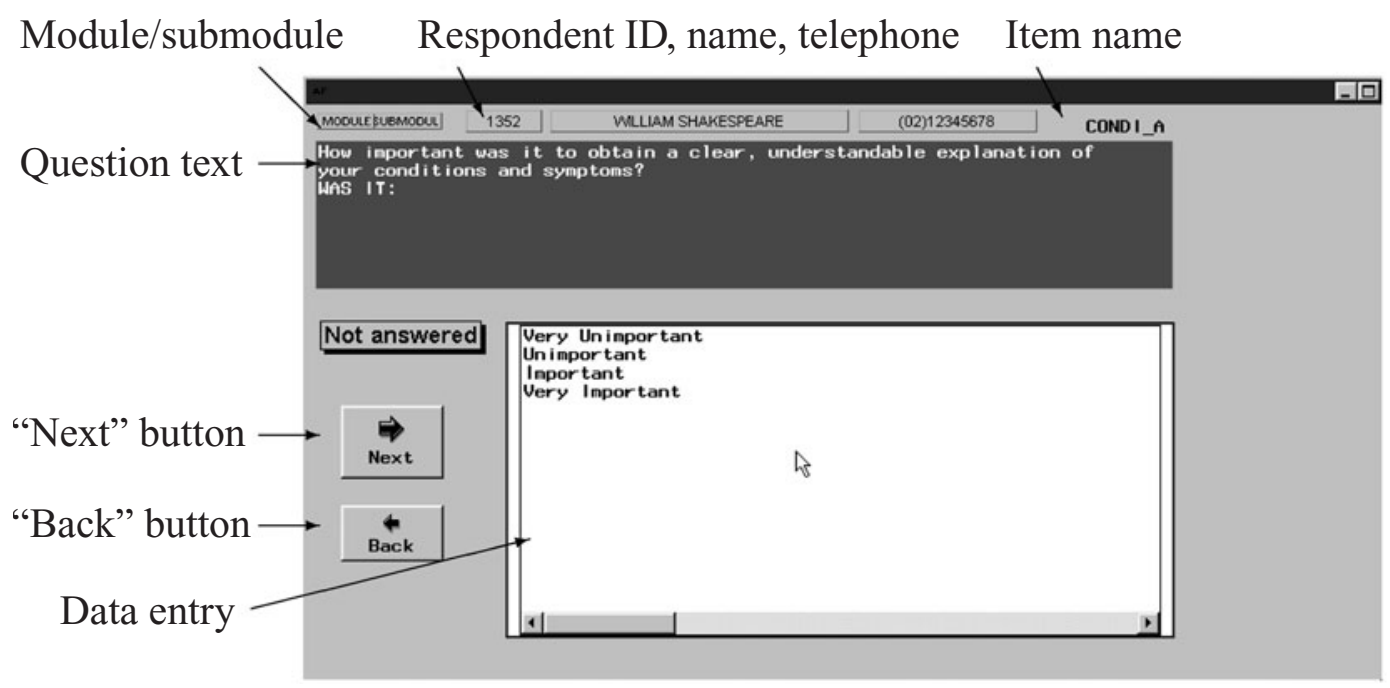

Figure 5. The GEIS interviewing window showing a question with four possible answers. The question was generated by a CHCE script item.

question to be redisplayed so that the previously entered answers might be corrected. In this way, interviewers might return as far back as the beginning of the interview. This was also possible in the IVR mode.

Other functions. GEIS produced reports to monitor data collection and interviewer performance. Once data were accumulated, they could be immediately displayed or analyzed using standard SAS programs. Respondent contact and interview control information could be edited, but not respondent answers. A backup facility allowed all project files to be saved together to another location.

\section{Implementing IVR Within GEIS}

To introduce IVR functions, additional hardware consisting of a voice card was required. The card allowed GEIS to initiate or receive calls, play recorded voice messages stored in files, record messages spoken by respondents, and respond to touchphone keypresses by the respondent. The voice recordings were stored in files that were specified in the script by the use of keywords.

When GEIS initiated a call to a household respondent, it needed to distinguish a person from an answering machine so it could respond correctly, using either acoustic analysis or salutation length determination. In acoustic analysis, the voice card used the quality of the sound to determine whether the line was answered by an answering machine. In the salutation length determination, the voice card monitored how long the respondent spoke when answering a call. For example, "hello" is briefer than "hello, we're not in at present." The first case is much shorter and would be assumed to be spoken by a person, whereas the longer second case would be assumed to be a recording on an answering machine. Since neither method was perfect, scripts had to be written to allow for the possibility of misidentification. These methods may improve as voice card technology develops.

\section{Selecting Interviewees}

Each survey might require a different method of selecting respondents for interview. To allow for this, GEIS used a series of variables, shown in Table 1, that combined to control how respondents were selected for an interview. Only those respondents for whom these variables contained appropriate values appeared in the CATI interviewer's pull-down list, so that those who had completed an interview were not recontacted, respondents who had arranged to complete the interview at a specific date and time would be called then (and not before or after), numbers that were not answered were called back later at intervals until contact was made, some numbers would not be called if a precontact letter was to arrive first, respondents known to be ineligible would not be called, and respondents who had refused would not be called back. The IVR system used an analogous method to select the respondents. Random digit dialing (Waksberg, 1978) is not currently implemented.

\section{Status Codes}

The STATUS variable stored the status of interviews as they progressed through contact attempts to interview finalization. The STATUS variable used a two-letter coding system, shown in Table 2 . The code was initially set to code NA (no attempt) and then typically progressed through various codes, as is shown in Table 3 . The code could not be changed from a higher degree of completion to a lower one; for example, it could not be changed from PQ (partly completed) to NA. Response rate calculations (American Association for Public Opinion Research, 2000) were done by calculating the proportions of various status codes (Corkrey \& Parkinson, 2002a).

An interrupted interview was saved with STATUS code PQ. These interviews were automatically resumed from the point left off when recontacted. When a fully 
Table 1

GEIS Variables Used to Select Interviewees

\begin{tabular}{cl}
\hline Variable & \multicolumn{1}{c}{ Function } \\
\hline START & $\begin{array}{l}\text { This variable was set to the earliest date and time a re- } \\
\text { spondent should be contacted. Respondents did not ap- } \\
\text { pear in the CATI interviewer's pull-down list and were } \\
\text { not available to the IVR system until the START date } \\
\text { and time had passed. }\end{array}$ \\
SELECTED* & $\begin{array}{l}\text { If the interviews for certain cases were to be prevented } \\
\text { indefinitely, this variable was set to zero. These respon- } \\
\text { dents would then not appear in the CATI interviewer's } \\
\text { pull-down list and were not available to the IVR sys- } \\
\text { tem. As soon as the variable was reset to one, the cases } \\
\text { became available immediately for interviewing. }\end{array}$ \\
ELIGIBLE & $\begin{array}{l}\text { Some cases could be removed because of ineligibility } \\
\text { by setting this variable to any positive value. These re- } \\
\text { spondents would not appear in the CATI interviewer's } \\
\text { pull-down list and were not available to the IVR sys- } \\
\text { tem until the ELIGIBLE value was reset to zero. }\end{array}$ \\
As interviews were contacted or completed or cases \\
STATUS \\
were eliminated from interviewing, their condition was \\
recorded using this variable.
\end{tabular}

*The SELECTED and ELIGIBLE variables performed the same function of selecting or deselecting respondents for interview but were used in different contexts. The SELECTED variable was used to select or deselect large numbers of respondents for interview, whereas the ELIGIBLE variable was used to select or deselect single respondents for interview for specific reasons. The SELECTED variable was principally set within SAS data step programs. An example was where respondents in one region were to be interviewed later in the survey than respondents in another region. The ELIGIBLE variable was principally set from the GEIS control panel. An example was where a respondent had refused or an information letter had been returned. In these cases, the ELIGIBLE variable would be set to an appropriate code.

completed interview ended, the status was set to the CQ code (completed questionnaire). However, if after having reached the end of an interview, the interviewer returned to an earlier item and changed an answer, GEIS would automatically reset the status back to PQ.

\section{Data Quality Considerations}

GEIS reduces inconsistencies and errors as a result of several factors.

Range limit checks. Many questions in scripts could require the entry of numeric data, such as a date of birth. In GEIS, numeric quantities could be entered as integers, numbers with decimals, dates, and times. Absolute limit checks and reasonableness limit checks consisted of a lower limit, an upper limit, or both. Numbers outside absolute limits could not be entered, whereas numbers outside the reasonableness limits had to be confirmed before being accepted. Limit checks were set within individual script items.

Item nonresponse recording. GEIS would not display the next question until the current question had been answered. This meant that answers to individual questions could not be omitted. However a respondent's re- fusal to answer a particular question could be recorded using a SAS special missing value: .R (SAS Institute Inc., 1990). The .R values were treated by default as missing values within ordinary SAS programs but could also be handled within GEIS scripts as any other value. Item nonresponse rates could be calculated as the proportion of groups of items with .R answers. If needed, other code values could be adopted for specific answer types.

Script logic checks. After a script was imported, GEIS compiled it into a format that it could use to generate the CATI or IVR interface. This process involved creating data sets and catalogues and extensively checking the script for errors. Compiler error and warning messages were written to the interviewing $\log$.

Workstation independence. Each CATI workstation stored all data locally until interview termination, when the data were uploaded to the share server. This allowed recovery following power failure or network interruption.

Programmatic constancy. Since no programming was required, any errors could be reliably ascribed to scripting errors. This particular aspect partly explained the very rapid interview development possible with GEIS (Corkrey, 2002).

GNU General Public License (GPL). To further improve the software quality, GEIS has been released under a GPL. Other users may correct bugs, extend it, or adapt it to local needs.

\section{IVR Call Scheduling}

Since the IVR system operated automatically, it used a call-scheduling algorithm (Kulka \& Weeks, 1988; Steel, Vella, \& Harrington, 1996) illustrated in Figure 6. Cases were assigned to four categories: callbacks due, answering machines, previously attempted noncontacts, and unattempted cases. Except for callbacks, calls were made at alternating short and long intervals. Typically, if no contact was made, a number would be rung 30 min later. Then, if no contact was made on the second attempt, it would be rerung after $8 \mathrm{~h}$ or even later. If still no contact was made, the number was rerung 30 min later, and so on.

Data Management

There were several GEIS data sets, shown in Table 4. A CONFID data set was created by the user to hold the

Table 2

Principal STATUS Codes

\begin{tabular}{ll}
\hline STATUS Code & \multicolumn{1}{c}{ Meaning } \\
\hline AM & answering machine \\
CB & callback arranged \\
CQ & completed questionnaire \\
DO & dropped partway, due to refusal \\
DR & dropped before starting, due to refusal \\
DT & disconnected tone \\
ER & error condition \\
ET & engaged tone \\
FM & fax machine \\
NA & no attempt made to contact \\
OS & out of scope-ineligible \\
PQ & partly completed interview \\
\hline
\end{tabular}


Table 3

Example of STATUS Codes in Successive Call Attempts

\begin{tabular}{clc}
\hline Call & \multicolumn{1}{c}{ Outcome } & STATUS Code \\
\hline 0 & & NA \\
& & $\downarrow$ \\
1 & Call rang out without answering. & RT \\
2 & Number was engaged. & $\downarrow$ \\
3 & Call answered by answering machine. & $\downarrow$ \\
& Respondent contacted and appointment & AM \\
4 & made to call back. & $\downarrow$ \\
& & $\downarrow$ \\
5 & Part-interview completed. & PQ \\
6 & Interview completed. & $\downarrow$ \\
\hline
\end{tabular}

respondent's details. When a script was imported, it was saved to the SCRIPT data set. During compilation, GEIS used information in the CONFID and SCRIPT data sets to create the other data sets. For each item in a script, GEIS created one or more variables in the ANSWERS data set, usually in the same order as in the script. The CONTROL data set contained the completion details of the interviews. There was an ID variable that acted as an index that linked all the data sets. The ID variable held numeric values that were unique for each respondent. All data sets were in SAS format.

During interviews, only a single interviewer could access a particular respondent's data at one time. This eliminated the possibility that two interviewers might make simultaneous attempts to ring the same respondent. To do this, when the interviewer selected a respondent, the respondent's ID value was used to locate the appropriate record in the data sets, and those records were then locked, using a share server (SAS Institute Inc., 1991), preventing their being modified by another user or program.

Any number of CATI simultaneous interviews could be conducted, but the voice card allowed only four at one time. Multiple simultaneous access to the data sets was

Table 4 The Major GEIS Data Sets

\begin{tabular}{cl}
\hline Name & \multicolumn{1}{c}{ Content } \\
\hline CONFID & $\begin{array}{l}\text { This data set contained the respondent contact details, in- } \\
\text { cluding name, telephone number, and address. } \\
\text { SCRIPT }\end{array}$ \\
$\begin{array}{l}\text { This data set was created when the script text file was im- } \\
\text { ported. It contained all information needed to define the } \\
\text { questionnaire, such as question texts, allowable options } \\
\text { for closed-form questions, range limit tests for numeric } \\
\text { questions, and the logical relationships between all the }\end{array}$ \\
items in the questionnaire.
\end{tabular}

controlled using record locking by means of a share server. The share server was an SAS program, run on a networked computer (see Figure 2), that allowed controlled access to data during collection, which was also useful for statistical analysis and monitoring programs.

\section{GEIS Scripts}

The GEIS script provided an open method of interview specification within multiple modes. It contained all the information needed to define the interview, including question types and texts, variable texts, option texts and codes, range limit information, question-branching information, status code information, calculations, do-loop information, and IVR information. These are specified by means of item types, self-protection statements (SPSs), and answer quoting.

Item types. A GEIS script consisted of a series of items corresponding to individual questions or performed other functions, such as sending e-mails. An example shown in Figure 7 defines a question about the respondent's education that allows seven possible answers.

A script item's first and second lines were used to specify various options. In the example (Figure 7), the item's type is CHCE and its name is Q2. A CHCE item type created a question with a restricted set of possible answers. The third line was an expression called the selfprotection statement (SPS), which will be explained later. In the example, this is Q1 NE. The next lines were used to specify the question text. In the example this is What is the highest level of schooling you have completed. Following this were settings specific to the item type or interview mode. In the example, this was a list of possible answers with numeric codes to be stored: $3 \mathrm{Sec}$ ondary school. The touchphone keys to be pressed in IVR mode could also be specified here. The penultimate line was used to specify the item's label assigned to the variable when the answer was saved. In the example, this is Education level. The last line was a line of asterisks that visually divided the script items from each other.

A complete list of item types is shown in the Appendix. GEIS automatically configured the interviewing system according to the item type and survey mode. As an example, a CHCE type within a CATI script caused GEIS to display the question and a list box containing the possible answers, of which only one could be selected (e.g., see Figure 5). In IVR, GEIS played the recorded questions and accepted only the specified code values.

Other item types (Appendix) were selected according to type of question to be asked or function required. Since there were very many items types, only a few are briefly described here. For example, a MULT type was used when asking a respondent to select zero, one, or more possible answers from a list of possible answers, whereas an OPEN item type allowed the respondent to enter any text or record a spoken message in IVR mode. Where a series of very similar items were to be asked, the DO and ENDD types were used to implement do-loops within a script. Any items between the DO and the ENDD items were repli- 


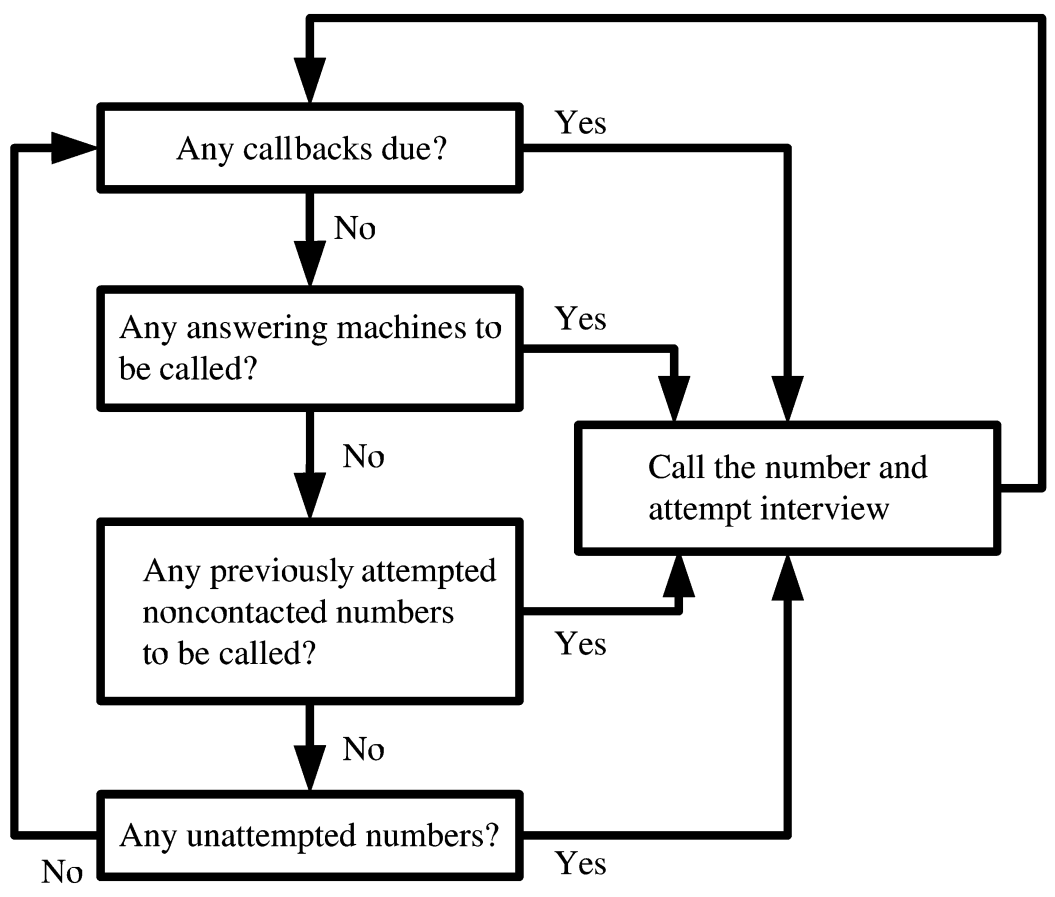

Figure 6. IVR call-scheduling loop.

cated a fixed number of times, and the item names and SPSs, described below, were adjusted as needed. The items within the do-loops could then use dynamically modifiable question texts that altered with each cycle around the loop. A very complex script could be broken down into subscripts, and these could be inserted by means of a SCRP item. External data could be imported using the LINK item type, either before the entire survey started or just before an interview started. These data could be used when dynamically altering question text or was evaluated within SPSs to control the logic of question flow. Lastly, a MAIL item could be used to send an e-mail once an interview had completed. For example, an e-mail could be used to send a recorded spoken comment made by a respondent within an IVR interview to a survey administrator.

The GEIS scripting method also allowed interview modes to be combined, such as the hybrid methods that combined CATI and IVR. This was done by simultaneously running two computers, one running a CATI script and another running an IVR script, and transferring calls between them as needed (Corkrey, 2002).

Self-protection statements. The order in which questions appeared in a script could depend on the answers given beforehand, or they may been asked in an unvarying question order. Also, CATI interviewers or IVR respondents could also move back several questions to a previ-

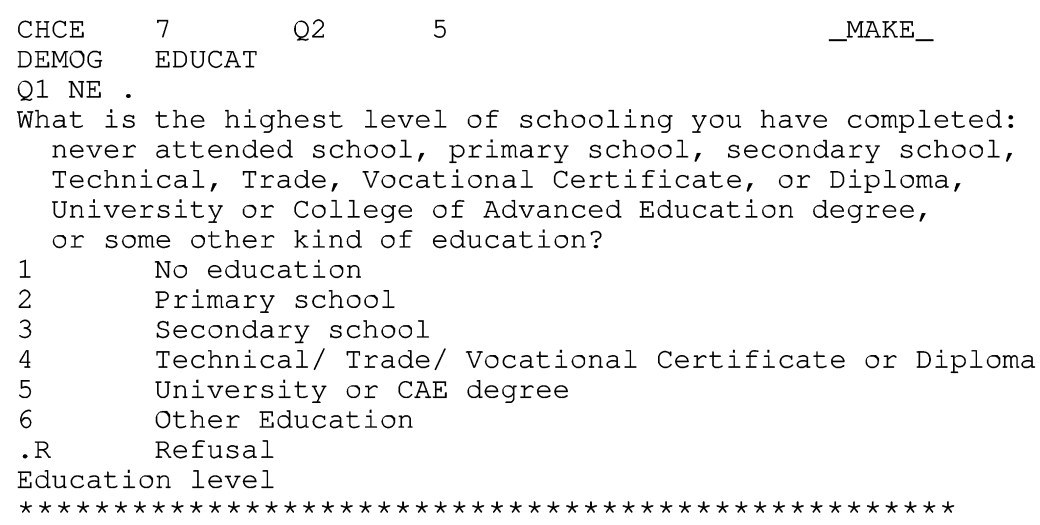

Figure 7. Example of a GEIS script item. This example shows a CHCE item. 
ously entered answer and alter it. The determination of which question to display in each case was done by SPSs. An SPS was so called because it determined whether a script item with which it was associated was protected or unprotected; if protected the item could not be displayed, and if unprotected the item could be displayed.

An SPS was a logical statement within each script item that could be either true or false. An SPS could make use of an answer to any preceding question by referring to that script item's name or could access external data by means of an previous LINK item. When an SPS was false, the associated item was deemed to be protected and could not be displayed. Conversely, if the SPS was true, the associated item was deemed unprotected and could be displayed. SPSs permitted the complex logic structure of a questionnaire to be condensed to simple statements.

In the example in Figure 8, the only allowable answers to Q1 are "male," coded as one, and "female," coded as two; Q2 is the same question as that shown in Figure 7 and accepts the codes 1, 2, 3, 4, 5, 6, and .R; Q3 accepts any numeric response; Q4 accepts "yes," coded as one, and "no," coded as two; and Q5 is omitted.

The SPS for Q2 is Q1 NE ., which refers to the answer given to question Q1. In the SPS, Q1 means the answer given to question Q1, NE means "not equal to," and "." (period) means "no answer." The effect of the SPS is that Q2 is unprotected and displayable only if Q1 has an answer. Similarly, the SPS for Q3 is Q2 NE ., which means that Q3 is unprotected and displayable only if Q2 has an answer. The SPS for item Q4 is (Q1 EQ 2) AND (Q3 NE .), in which "EQ" means "equal to" and "AND" means that both the parenthetical expressions must be true for the whole SPS to be true. This SPS causes the Q4 item to be displayed only if the answer to Q1 is "female" and Q3 is answered. The last item, Q5, may be displayed only if item Q3 or Q4 has been answered. Overall, male respondents would be asked questions Q1, Q2, Q3, and Q5, and female respondents would be asked all five questions. Note that the SPSs, such as those for Q4 and Q5, can refer to items at any earlier point in the script.

The SPSs were constructed by the user to allow each respondent a single path through the script. When the script was compiled, GEIS automatically detected logical errors in the SPSs, such as where it was not possible to jump forward from an item or where it was not possible to reach an item. As data were entered, GEIS automatically updated and evaluated the script SPSs. GEIS then allowed the interviewer only to move backward or forward to the first unprotected item. In this way, the sequence of questions asked was determined by the SPSs and data previously entered.

Answer quoting and calculations. Question texts could be modified dynamically within any item during the progress of an interview. An example would be the question, "Is the telephone number XXXX XXXX the correct number to ring?" This process was termed answer quoting and usually consisted of inserting the answers to earlier questions into the current question's text, but data

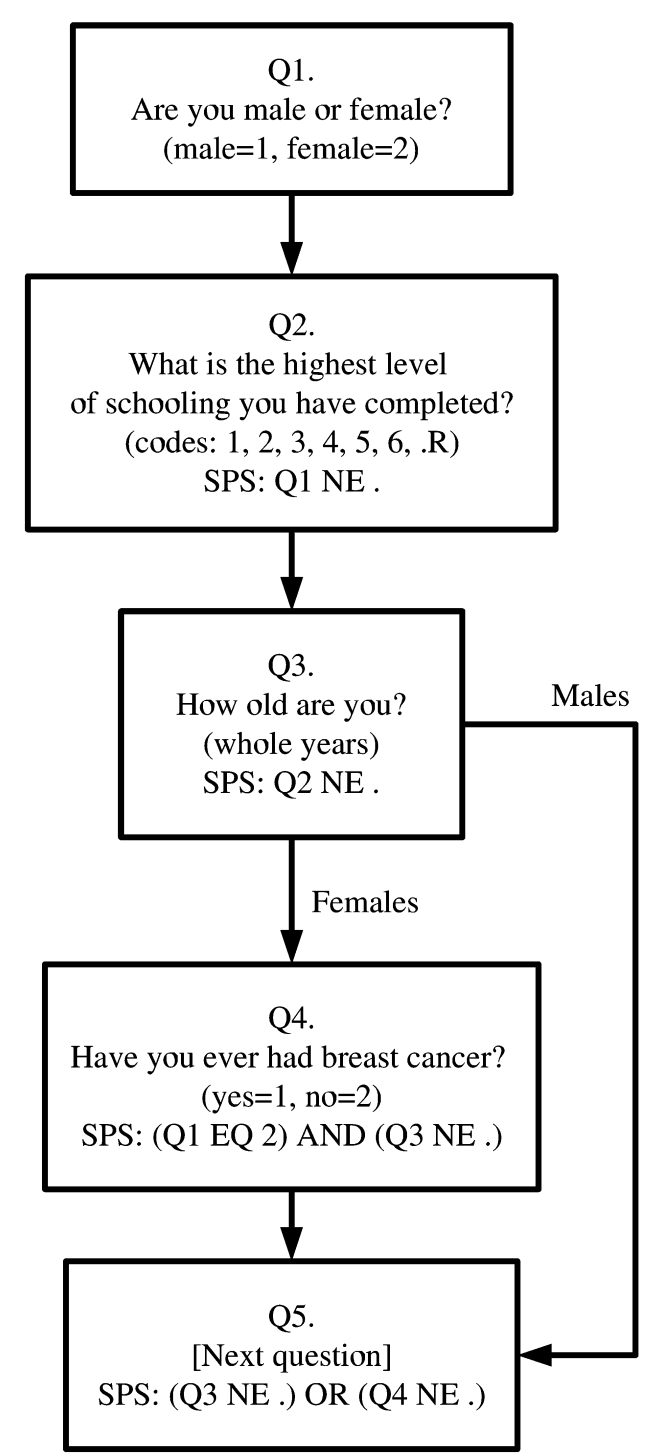

Figure 8. Example of the logical relationships between five questions, their self-protection statements, and option-code values. NE means "not equal to," and "." (period) indicates a missing answer. For example, the SPS Q1 NE . indicates that Q2 may not be displayed until $Q 1$ has an answer.

from external sources could also be used. To quote an answer to a previous item, the previous item's name was inserted in the script item's question text and surrounded by carets. For example, Is the telephone number ${ }^{\wedge} Q 4^{\wedge}$ the correct number to ring? where Q4 contained the telephone number. Another example is the dynamic creation of e-mail text during the interview.

Simple arithmetic operations could also be defined, such as ${ }^{\wedge} \mathrm{Q} 5-3^{\wedge}$, which subtracted 3 from the value of Q5 and then inserted the formatted value into the question text. For more complex calculations, a CALC item allowed any data manipulation SAS could perform to be done during the interview. A typical use was searching databases. 


\section{An Application of GEIS}

As has been described elsewhere (Corkrey \& Parkinson, 2002a), GEIS was used to conduct an Australia-wide telephone survey of households in 2000 . Herein is described a brief comparison of the results with those of an independent survey.

Method. A total of 3,100 households were selected, covering all the states and territories of Australia. Four different telephone interview methods were used: CATI, IVR, and two combination methods: Hybrid I, in which the interview began with CATI and only the more sensitive questions were asked using IVR, and Hybrid II, in which only IVR was used for all the questions except the introduction. No remuneration was offered. Simple instruction sheets showing a picture of a typical touchphone keypad were included with letters mailed in advance.

All the computers used were Pentium II computers. A four line Dialogic $\mathrm{D} / 41 \mathrm{H}$ voice card was installed in one computer to handle the IVR functions. Seven telephone lines were used, three for the interviewers and four for the IVR system. In the hybrid methods, interviewers transferred calls to the IVR system after contacting and recruiting respondents. Voice recordings were made in 16-bit monaural $11-\mathrm{kHz}$ format with an Optimus 333104 omnidirectional microphone and Creative Sound Blaster Vibra 128 sound card. Voice recordings were by a single female staff member selected using a voice assessment method based on Oksenberg, Coleman, and Cannell (1986).

The interview addressed the respondent's consumption of alcohol and drugs and ended with demographic questions. The scripts can be downloaded from the Psychonomic Society's archival depository. Answers to open-ended questions were entered verbatim by CATI interviewers, whereas IVR respondents could record short spoken sentences as their responses. For numeric answers, absolute and reasonable limits prevented range errors. Invalid numeric responses triggered an appropriate message or display. In all the interview methods, respondents could refuse to answer a particular item, and if they wished, they could return to earlier questions and modify their answers.

To provide a comparison, the National Drug Strategy Household Survey (NDSHS; Australian Institute of Health and Welfare, 1999) was used. This was a biennial household survey of alcohol and drug use that used a multistage stratified sample design and face-to-face interviews collecting a total of 10,030 interviews. The more sensitive questions were asked using confidential self-administered questionnaires, which were sealed in envelopes by the respondents prior to handing them to the interviewer.

Drinking status obtained by each method was compared with the NDSHS (Adhikari \& Summerhill, 2000) results after the data were weighted by age and sex according to the ABS Census data (Australian Bureau of Statistics, 1996).

Results. The contact rates $(84 \%-86 \%)$ of all the methods and the response rates $(56 \%-61 \%)$ for CATI, Hybrid I, and Hybrid II did not differ significantly, but IVR ob- tained a significantly lower response rate than did CATI $(12 \%$ vs. $61 \% ; p<.001)$.

Since the distributions of drinking status did not differ between Hybrid I and Hybrid II for males $\left[\chi^{2}(3)=\right.$ $3.0, p=.40 ; n=200]$ or females $\left[\chi^{2}(3)=2.6, p=.45\right.$; $n=324]$, the data from the two methods were combined. As is shown in Table 5, the hybrid and the IVR methods did not differ significantly from the NDSHS results with respect to drinking status. However, the CATI method differed significantly from the NDSHS for both males and females. CATI female respondents underreported being ex-drinkers and overreported never having drank, whereas male respondents overreported being regular drinkers.

Discussion. All the methods contacted similar proportions of their respective samples, but the IVR method had fewer respondents. The combined hybrid method results agreed with the NDSHS, whereas CATI deviated in the direction expected for sensitive questions. Due to a small sample size, the results for the IVR method were considered unreliable. By assuming that the NDSHS was a gold standard, the hybrid methods provided more accurate telephone survey data on alcohol consumption than did CATI alone.

\section{Conclusions}

GEIS provides an open and flexible method for scripting CATI and IVR interviews without the need for programming, resulting in rapid interview development, a controlled interviewing environment, and built-in data

Table 5

Drinking Status by Method for Males and Females, Compared With the NDSHS Results

\begin{tabular}{|c|c|c|c|c|c|}
\hline \multirow[b]{2}{*}{ Method } & \multirow[b]{2}{*}{ Sex } & \multicolumn{2}{|l|}{ Drinking } & \multicolumn{2}{|l|}{ NDSHS } \\
\hline & & Status & $\% *$ & $(\%)$ & $p \dagger$ \\
\hline \multirow[t]{8}{*}{ CATI } & Female $(n=233)$ & Regular & 39 & 39 & $<.01$ \\
\hline & & Occasional & 33 & 39 & \\
\hline & & Ex-drinker & 7 & 11 & \\
\hline & & Never drank & 21 & 12 & \\
\hline & Male $(n=133)$ & Regular & 72 & 59 & .03 \\
\hline & & Occasional & 19 & 25 & \\
\hline & & Ex-drinker & 5 & 9 & \\
\hline & & Never drank & 4 & 7 & \\
\hline \multirow[t]{8}{*}{ Hybrid $\ddagger$} & Female $(n=301)$ & Regular & 39 & 39 & .62 \\
\hline & & Occasional & 40 & 39 & \\
\hline & & Ex-drinker & 11 & 11 & \\
\hline & & Never drank & 10 & 12 & \\
\hline & Male $(n=186)$ & Regular & 59 & 59 & .63 \\
\hline & & Occasional & 28 & 25 & \\
\hline & & Ex-drinker & 7 & 9 & \\
\hline & & Never drank & 6 & 7 & \\
\hline \multirow[t]{8}{*}{ IVR } & Female $(n=53)$ & Regular & 39 & 39 & .37 \\
\hline & & Occasional & 38 & 39 & \\
\hline & & Ex-drinker & 17 & 11 & \\
\hline & & Never drank & 6 & 12 & \\
\hline & Male $(n=24)$ & Regular & 54 & 59 & .52 \\
\hline & & Occasional & 20 & 25 & \\
\hline & & Ex-drinker & 16 & 9 & \\
\hline & & Never drank & 10 & 7 & \\
\hline
\end{tabular}

*The data were weighted by age and sex according to the ABS Census data (Australian Bureau of Statistics, 1996). †Goodness-of-fit $p$ values. $\ddagger$ Combined Hybrid I and Hybrid II methods. 
quality checks. It is suggested that GEIS represents a standard means of interview specification for CATI and IVR. Further development is needed to support additional modes, an open data storage format (e.g., XML; Archard, Vaysseix, \& Barillot, 2001), and user-friendly front-ends for script generation. We also see no hindrance to including support for Web-based and e-mail surveys, which may then be combined with the CATI and IVR modes. This may assist in overcoming the restrictions currently extant in Web-based and e-mail surveys, such as poorly defined frames and variable response rates (Schaefer \& Dillman, 1998; Sheehan \& Hoy, 1999; Smith, 1997), and ensuring that sensitive questions are asked using the more anonymous modes. It has been shown that GEIS may be used to reliably conduct CATI, IVR, or combined mode surveys.

\section{REFERENCES}

ADHIKARI, P., \& SUMmerhill, A. (2000). 1998 National Drug Strategy: Household survey. Detailed findings (Drug Statistics Series No.6, AIHW catalogue No. PHE 27). Canberra: Australian Institute of Health \& Welfare.

Alemagno, S. A., Cochran, D., Feucht, T. E., Stephens, R. C., Butts, J. M., \& Wolfe, S. A. (1996). Assessing substance abuse treatment needs among the homeless: A telephone-based interactive voice response system. American Journal of Public Health, 86, 1626-1628.

American Association for Public Opinion Research (2000). Standard definitions: Final dispositions of case codes and outcome rates for surveys. Lenexa, KS: Author.

ARChaRD, F., VAYsSEIX, G., \& BARILlot, E. (2001). XML, bioinformatics and data integration. Bioinformatics Review, 17, 115-125.

Artisoft Inc. (1998). Visual Voice Pro (Version 5.0) [Computer software]. Author.

Australian Bureau of Statistics (1996). 1996 Census of population and housing: Basic community profiles. In AusStats [electronic resource]. Available at http://www.abs.gov.au/ausstats. Canberra: Author.

Australian Institute of Health and Welfare (1999). National drug strategy: Household survey. Technical Report 1998. Canberra: Australian Institute of Health \& Welfare, Department of Health \& Aged Care.

Cannell, C. F., \& Fowler, F. J. (1963). Comparison of a selfenumerative procedure and a personal interview: A validity study. Public Opinion Quarterly, 27, 250-264.

Corkrey, R. (2002). Exploring the use of interactive voice response as a population health tool. Ph.D. thesis, University of Newcastle. Available at http://www.newcastle.edu.au/services/library/adt/index.html.

CORKREY, R., \& PARKINSON, L. (2002a). A comparison of four computerbased telephone interviewing methods: Getting answers to sensitive questions. Behavior Research Methods, Instruments, \& Computers, 34, 354-363.

Corkrey, R., \& Parkinson, L. (2002b). Interactive voice response: Review of studies 1989-2000. Behavior Research Methods, Instruments, \& Computers, 34, 342-353.

Couper, M. P., Traugott, M. W., \& Lamias, M. J. (2001). Web survey design and administration. Public Opinion Quarterly, 65, 230-253.

Da-Silva, C. Q., Rodrigues, J., Leite, J. G., \& Milan, L. A. (2003). Bayesian estimation of the size of a closed population using photo-id with part of the population uncatchable. Communications in Statistics, 32, 677-696.

HANSEn, M. S., \& Dørup, J. (2001). Wireless access to a pharmaceutical database: A demonstrator for data driven Wireless Application Protocol (WAP) applications in medical information processing [On line]. Journal of Medical Internet Research, 3, e4.

Kissinger, P., Rice, J., Farley, T., Trim, S., Jewitt, K., Margavio, V., \& MarTin, D. H. (1999). Application of computer-assisted interviews to sexual behavior research. American Journal of Epidemiology, 149, 950-954.
Kulka, R. A., \& Weeks, M. F. (1988). Towards the development of optimal calling protocols for telephone surveys: A conditional probabilities approach. Journal of Official Statistics, 4, 319-332.

NiCHOLLS, W. L., II (1988). Computer-assisted telephone interviewing: A general introduction. In R. M. Groves, P. P. Biemer, L. E. Lyberg, J. T. Massey, W. L. Nicholls, II, \& J. Waksberg (Eds.), Telephone survey methodology (pp. 377-385). New York: Wiley.

Oksenberg, L., Coleman, L., \& Cannell, C. F. (1986). Interviewers' voices and refusal rates in telephone surveys. Public Opinion Quarterly, 50, 97-111.

SAS Institute INC. (1990). SAS Language: Reference, Version 6 (1st ed.). Cary, NC: Author.

SAS InSTITUTE INC. (1991). SAS/SHARE Software: Usage and Reference, Version 6 (1st ed.). Cary, NC: Author.

SAS InSTITUTE INC. (1999). SAS/STAT User's Guide, Version 6 (4th ed., Vol. 1 and 2). Cary, NC: Author.

Schaefer, D. R., \& Dillman, D. A. (1998). Development of a standard e-mail methodology. Results of an experiment. Public Opinion Quarterly, 62, 378-397.

SheeHan, K. B., \& Hoy, M. G. (1999). Using e-mail to survey internet users in the United States: Methodology and assessment. Journal of Computer-Mediated Communication [On line], 4. Available at http://www.ascusc.org/jcmc.

SMITH, C. B. (1997). Casting the net: Surveying an internet population. Journal of Computer-Mediated Communication [On line], 3. Available at http://www.ascusc.org/jcmc.

Steel, D., Vella, J., \& Harrington, P. (1996). Quality issues in telephone surveys: Coverage, non-response and quota sampling. Australian Journal of Statistics, 38, 15-34.

WAKSBERG, J. (1978). Sampling methods for random digit dialing. Journal of the American Statistical Association, 73, 40-46.

\section{ARCHIVED MATERIALS}

The following materials associated with this article are retrievable from the the Psychonomic Society's Norms, Stimuli, and Data archive Web archive, http://www.psychonomic.org/archive.

To access the above files or links, please visit http://www.psychonomic. org/archive/ and search for this article using the journal (Behavior Research Methods, Instruments, \& Computers), the first author's name (Corkrey) and the publication year (2004).

DIRECTORY: geis $\backslash$ binl.

DESCRIPTION: A directory containing the main program executables and syntax file (geissynx.txt).

DIRECTORY: geis $\backslash$ doclexamples\.

DESCRIPTION: A directory containing various small script examples. DIRECTORY: geis \doc \geisdemol.

DESCRIPTION: A directory containing a complete CATI survey example and script (geisdemo.txt).

DIRECTORY: geis $\backslash$ doc $\backslash$ server $\backslash$ sas share Server.sas.

DESCRIPTION: Contains an example of a program to run a share server.

DIRECTORY: geis $\backslash$ doc $\backslash$ geis.pdf.

DESCRIPTION: Contains the GEIS manual.

DIRECTORY: geisletclcommon〉.

DESCRIPTION: A directory containing SAS data sets to hold information common to various survey projects.

DIRECTORY: geisletclinitl.

DESCRIPTION: A directory containing SAS programs used in setting up interviewing workstations.

DIRECTORY: geis $\backslash$ COPYING.TXT.

Description: Contains the GNU General Public License that must accompany all copies of the program.

DIRECTORY: geis $\backslash$ README.TXT.

DESCRIPTION: Contains a general introductory note.

DIRECTORY: geis \run_geis.sas.

DESCRIPTION: Contains a demonstration program to run after the installation is complete.

AUTHOR's E-MAIL ADDRESS: r.corkrey@abdn.ac.uk. 


\section{GEIS Script Item Types (Functions Were Identical for CATI and IVR Scripts Except Where Indicated Otherwise)}

\begin{tabular}{ll}
\hline Item & \multicolumn{1}{c}{ Function } \\
\hline CALC & $\begin{array}{l}\text { CALC inserted a calculation into the script that was } \\
\text { executed when the item became active. The calcula- } \\
\text { tion might involve the value of any previous answer } \\
\text { or imported values. They might only be simple ex- } \\
\text { pressions, such as Q2 }+3 \text {, but could also be very } \\
\text { complex. }\end{array}$
\end{tabular}

CALL When a CALL item became active in an interview, the telephone number of the respondent stored in the variable STDPHONE was called.

CBDT This item stored the date that the respondent should be called back.

CBTM This item stored the time that the respondent should be called back.

CHCE This item was used to ask a question that had only a limited set of possible answers, such as "yes" and "no." In CATI mode, GEIS displayed a list box with between 1 and 50 text options, one of which might be selected by the interviewer. In IVR mode, GEIS allowed the respondent to choose one option by pressing one of the keys $1,2, \ldots, 9$ on the touchphone.

DO This item started a do-loop. A do-loop might contain a series of other items that were executed multiple times. For example, the following questions could be replaced by a single item (not shown) within a doloop.

"Has your oldest child been immunized?"

"Has your second oldest child been immunized?" "Has your third oldest child been immunized?"

ENDD This item ended a do-loop.

HUP In CATI mode or IVR mode, this item hung up a telephone call and, optionally, returned the call to another extension.

INFO In CATI mode, this item displayed up to 12 lines of text on the screen. Additional lines could be scrolled into view. It was used to give instructions to the interviewer or to display text to be read out to the respondent. In IVR mode, this item played a message. After the message had played, GEIS immediately activated the next item, paused briefly before moving on, paused indefinitely until a key was pressed, paused until a particular key was pressed, or paused until a certain time elapsed.

LINK This item imported one or more variables from another data set. The data were imported during compilation but could also be updated immediately before an interview.

LIST This item was identical to a CHCE item, except that the number of options that could be selected was unlimited. The options had to be specified in an external data set. A typical question was

"In which country were you born?"

for which there could be very many possible answers. This item was not implemented in IVR mode.

LVLC This item counted how many of a range of CHCE or MULT items in the script had had a specified option selected. For example, it may be used to check whether all of a set of CHCE questions were answered with "yes."

\begin{tabular}{ll} 
Item & \multicolumn{1}{c}{ Function } \\
\hline MAIL & This item sent an e-mail after the interview was com- \\
& pleted. The address of the recipient, cc address, sub- \\
ject, and attachment, were specified in other vari- \\
ables.
\end{tabular}

MULT This item was used to ask a question that could have zero, one, or more possible answers. In CATI mode, GEIS displayed a list box with between 1 and 50 text options, 1 or more of which could be selected by the interviewer. An example of a typical question is

"Which of the following diseases have you had: Influenza,
Measles,
Chicken pox?'

$$
\text { Chicken pox?" }
$$

This item was not implemented in IVR mode.

NEW If this item was activated during an interview, it appended a new respondent record to the main data sets.

NULL This item was used to link together logic paths in a script. It enabled scripts to be simplified by breaking up lengthy SPSs into several shorter ones that were shared between several NULL items.

NUM The NUM item was used to ask a question that required a numeric answer. The answer could be any valid SAS numeric value. Numbers (e.g., 1, 2, 3, -7.1, 0.03), dates (e.g., 23jan1990, 230199, OCT3), and times (e.g., 12:40PM, 17:00, or 9:00:40), could be entered. Upper and lower reasonableness and absolute limits could be specified. If the interviewer entered a value outside the reasonableness limits, GEIS displayed a check box that had to be ticked before another item would be displayed. If the interviewer entered a value outside the absolute limits, GEIS displayed an error message and refused to accept the answer. With IVR mode, dates and times had to be entered using digits only. For example, the date 23Jan 1950 was entered as 23011950. Upper and lower reasonableness limits could be specified within the IVR mode as well. If the respondent entered a value outside the reasonableness limits, GEIS played a message asking the respondent to confirm the value by pressing the hash key before the next item would be played. If the respondent entered a value outside the absolute limits, GEIS played an error message, and the question was repeated.

NUMM This item was similar to the NUM field but allowed up to five entry fields. An algebraic expression was used to set the value of a summary variable. A constraint expression ensured the field values were consistent. This item was not implemented in IVR mode.

OPEN This item was used to ask a question that required an open-ended answer. In CATI mode, the interviewer typed the verbatim answer into a text box. In IVR mode, the respondent was asked to speak their answer, which was then recorded by GEIS.

RST If this item became active, the interview was reset, all data were erased, and the first item then became active again.

SCAL This item was used to define one or more numeric or character values. Although a LINK item could be 
APPENDIX (Continued)

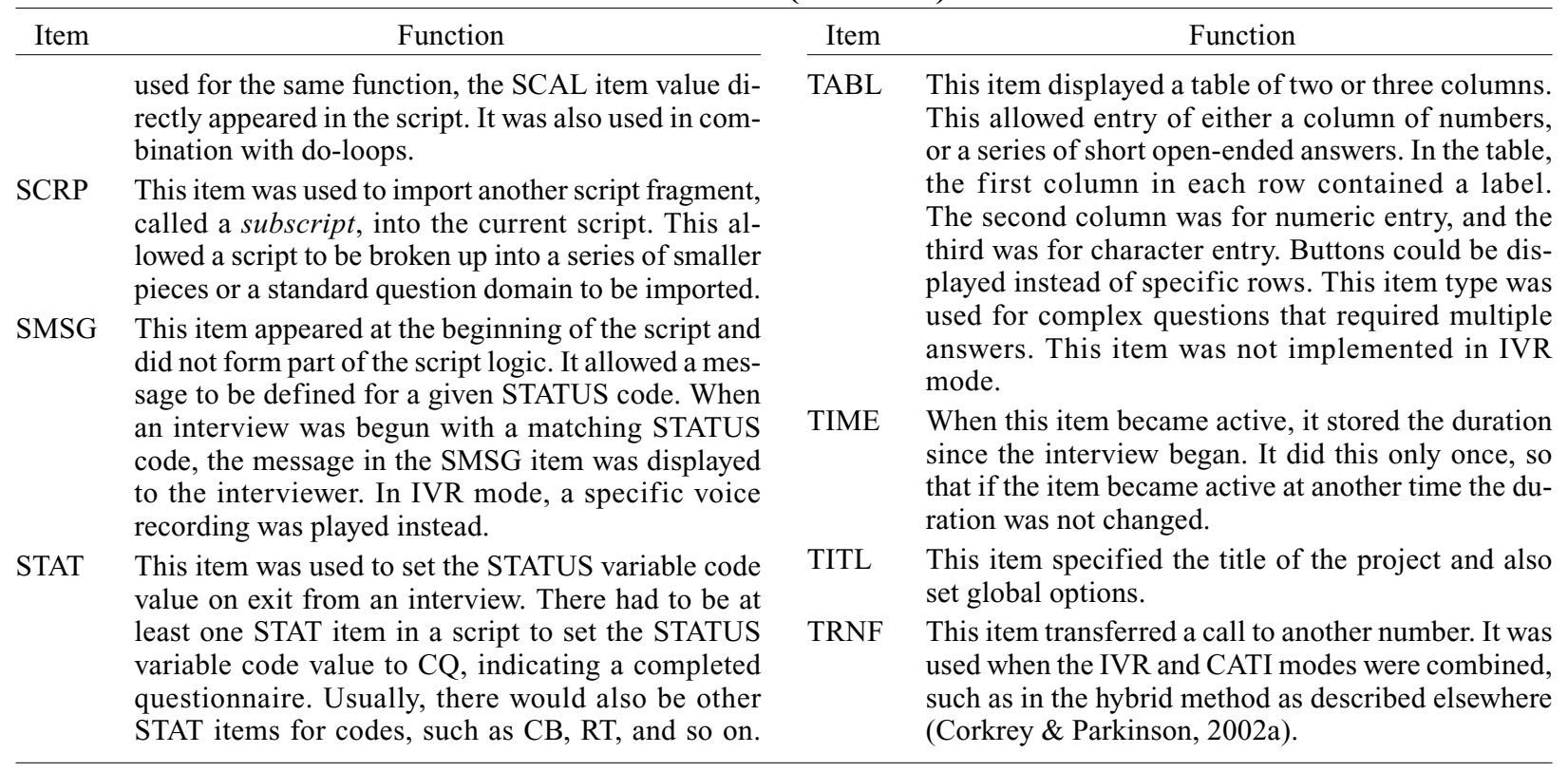

(Manuscript received January 7, 2003;

revision accepted for publication June 4, 2004.) 